\title{
Physical Education Motion Correction System Based on Virtual Reality Technology
}

\author{
$\underline{\text { https://doi.org/10.3991/ijet.v14i13.10710 }}$ \\ Yongzhong Yang $\left({ }^{(}\right)$ \\ Yangtze Normal University, Chongqing, China \\ 9123055 @qq. com \\ Lingfeng Meng \\ Hebei Sport University, Shijiazhuang, China
}

\begin{abstract}
Compared with traditional physical education (PE), the computer virtual digital-based education system is convenient and efficient, which can effectively solve the shortcomings of traditional teaching mode, and correct the PE motions in real time. Thus, the athletes can master the motion technical skills as soon as possible and the training effect can be improved. For this, based on computer virtual reality technology, this paper constructs a PE motion correction system. Then, through the analysis for real-time motion data and human movement posture, it achieves the training objective of real-time PE teaching motion correction under the three-dimensional visualization. This shall provide a scientific basis for the evaluation of $\mathrm{PE}$ teaching and training methods.
\end{abstract}

Keywords - Virtual reality, motion capture, physical education (PE), motion correction

\section{$1 \quad$ Introduction}

With the reform of PE curriculum in colleges and universities, the computer virtual reality technology has been adopted to break the shackles of the traditional rigid curriculum framework, and convert the teaching system from the two-dimensional world to the virtual three-dimensional world, so as to significantly change the educational form and environment, and the basic elements of the educational process as well as their mutual relationships [1]. The traditional PE teaching method is mainly based on subjectivity and experience. The actual practice experience of the coach directly affects the learning progress of the athletes, which makes the traditional physical education teaching start from the scientific visualization environment of virtual reality or augmented reality, and greatly expands the activity space of PE teaching [2-3]. So, in the process of PE training, the PE teaching motion correction should be made in real time, so that athletes can master the essentials of motion technology as soon as possible, reduce blind repetition and improve training effect [4]. For many years, China's $\mathrm{PE}$ teaching has been exploring to improve [5]. The computer-aided teaching in PE is 
computer-aided teaching system mostly in two-dimensional space such as text, sound, graphics, image and video, and most of them are mainly listening and watching, but lacking 3D real-time interaction and feed-back [6-7].

In recent years, domestic and foreign research on human motion capture in virtual reality technology has achieved advanced scientific research results [8]. Researchers have developed an application system based on human motion and visualization to facilitate the classification of motion movements [9]. The computer virtual technology is used to monitor the motion characteristics in real time, assist the coach to judge the standard level of the athlete's physical movement in time, analyse the movement situation through the virtual reality scene in $3 \mathrm{D}$ visualization and correct the $\mathrm{PE}$ movement in real time [10]. Also, the computer virtual reality technology is applied to capture the training movements of PE, obtain the related PE parameters and index parameters, while the real-time motion data and human sports motion posture are analysed to realize the teaching objective of real-time PE motion correction based on three-dimensional visualization [11]. Thus, the timely diagnosis and analysis of PE movements or training movements should be conducted to improve the training content and intensity purposefully, in order to carry out PE in a targeted manner, and ensure the scientific and digitization of PE training [12].

\section{Virtual Motion Based on Motion Capture}

\subsection{Architecture of virtual display system}

The design and implementation of PE motion correction system based on computer virtual technology requires the virtual display application platform to realize real-time three-dimensional dynamic database and virtual reality demonstration environment [13-14]. In this paper, one two-channel rear-projection stereo display system was adopted, where workstation computer 1 is responsible for receiving users' interactive information and data calculation, and the workstation computer 2 is for graphic calculation and drawing. The two workstation computers transmit data through the network, and then fuse the calculation results into the projector through the data line for image correction and edge fusion calculation; then, the images are respectively transported to four projectors, while each two projectors form a group to form a twochannel stereoscopic display system, and achieve the three-dimensional effect by polaroid stereoscopic glass-es [15-16]. The machines and devices are connected to each other through a network and a serial cable to realize coordinated control and display. Fig. 1 shows the structure diagram of the rear projection display system. 


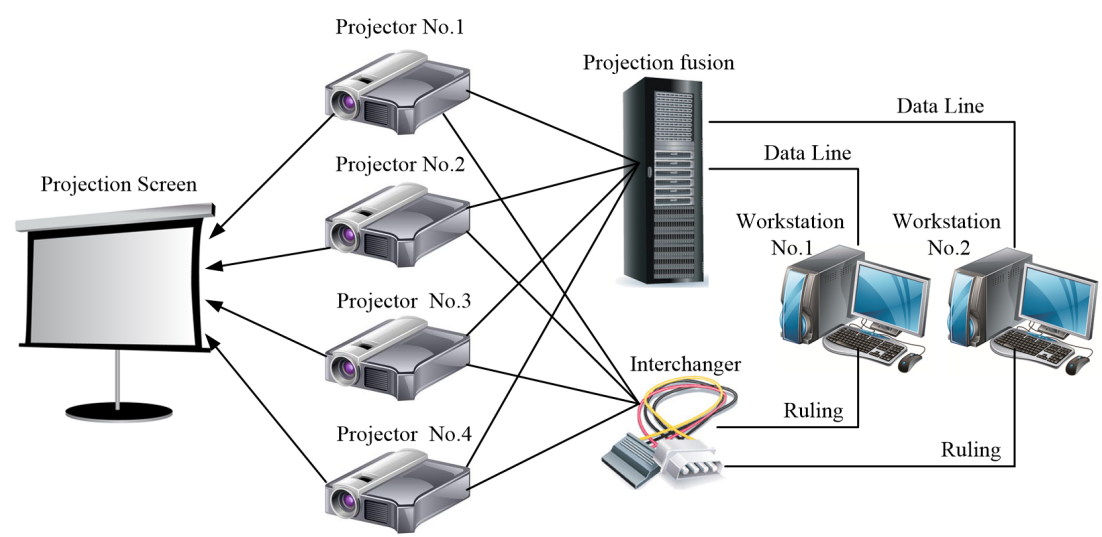

Fig. 1. The architecture diagram of rear projection display system

\subsection{Algorithm and implementation}

To correct the computer virtual technology-based PE teaching action, it is necessary to construct a virtual reality system, and make real-time three-dimensional visual interaction of sports movements [17-18]. Firstly, the coordinate system transformation of the motion capture system and the motion relationship between various parts of the human body should be solved. If the human body is simplified into a multi-rigid system, its motion form shall be a multi-body dynamics and kinematics solution process [19]. In order to strengthen the authenticity of the PE virtual reality system, the human body mathematical model and the three-dimensional geometry are combined to create a human kinematic behaviour model with physical properties, which is integrated into the PE virtual reality system through the VC++, ODE and APIs of Vega Prime. The physics engine open source code in conjunction with $\mathrm{VC}++$ is used for secondary development [20].

The solving method of multi-body dynamics mainly includes the Lagrangian analytical mechanical equation [21-22]. Firstly, the rectangular Cartesian coordinates are used to create the reference coordinate system, that is, the geodetic coordinate system, and the unit vector is defined as $\vec{e}_{o}=\left(\vec{e}_{o x}, \vec{e}_{o y}, \vec{e}_{o z}\right)$. Then, for the simplified human multiple rigid body, each tissue is simplified into a rigid local coordinate system $S_{i}$, and its unit vector is defined as $\vec{e}_{i}=\left(\vec{e}_{i x}, \vec{e}_{i y}, \vec{e}_{i z}\right)$. Finally, the unit vector of the rigid body is aligned with the local coordinate system of the rigid body, and the origin coordinates, displacement vector and rotation matrix $C_{o i}(3 \times 3)$ of the rigid body $S_{i}$ 's centroid position are defined according to the unit vector of the reference coordinate system as shown in the following: 


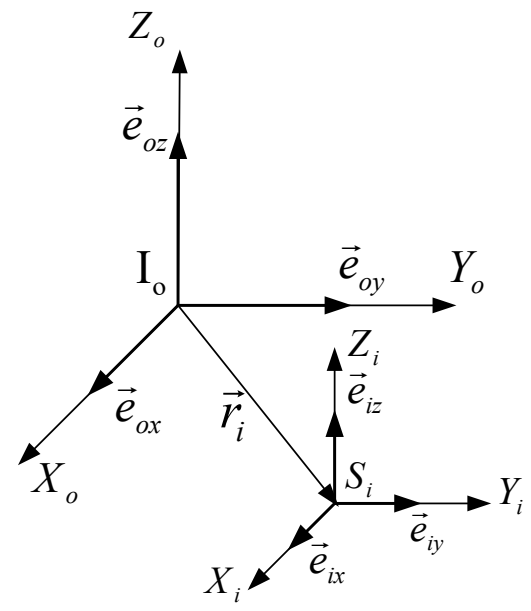

Fig. 2. Displacement vector coordinate system from reference system to rigid body center of mass

According to Fig. 2, the displacement vector $\vec{r}_{i}(3 \times 1)$ is defined by the rectangular coordinate system at the rigid body centroid position, and represented by a column matrix in the geodetic reference coordinate system, as shown in formula 1. The rotation matrix $C_{o i}(3 \times 3)$ is defined by the element matrix rotating around the coordinate axis and the Euler/Cardan angle $\phi=\left[\begin{array}{lll}\alpha & \beta & \gamma\end{array}\right]^{T}$, as shown in formula 2.

$$
\begin{aligned}
& \vec{r}_{i}(3 \times 1)=\left(\begin{array}{lll}
r_{i x / o} & r_{i y / o} & r_{i z / o}
\end{array}\right)\left(\begin{array}{l}
\vec{e}_{o x} \\
\vec{e}_{o y} \\
\vec{e}_{o z}
\end{array}\right)=r_{i x / o} \vec{e}_{o x}+r_{i y / o} \vec{e}_{o y}+r_{i z / o} \vec{e}_{o z} \\
& C_{o i}(3 \times 3)=\left[\begin{array}{ccc}
\cos \beta \cos \gamma & \sin \beta \\
\sin \alpha \sin \beta \cos \gamma+\cos \alpha \sin \gamma & -\sin \alpha \sin \beta \sin \gamma+\cos \alpha \cos \gamma & -\sin \alpha \cos \beta \\
-\cos \alpha \sin \beta \cos \gamma+\sin \alpha \sin \gamma & \cos \alpha \sin \beta \sin \gamma+\sin \alpha \cos \gamma & \cos \alpha \cos \beta
\end{array}\right]
\end{aligned}
$$

Thus, the six degrees of freedom of the rigid body $S_{i}$ in space are described by generalized coordinates (Formula 3 ). The matrix $v_{i}$ of velocity vector at centre of rigid body $S_{i}$ and the angular velocity vector matrix $\theta_{i}$ are shown in Formula 4 and 5 .

$$
\begin{aligned}
& P_{i}=\left[\begin{array}{llllll}
r_{i x / o} & r_{i y / o} & r_{i z / o} & \alpha_{i} & \beta_{i} & \gamma_{i}
\end{array}\right]^{T} \\
& v_{i}=\left[\begin{array}{l}
\dot{r}_{i x / o} \\
\dot{r}_{i y / o} \\
\dot{r}_{i z / o}
\end{array}\right]
\end{aligned}
$$




$$
\theta_{i}=\left[\begin{array}{ccc}
1 & 0 & \sin \beta \\
0 & \cos \alpha & -\sin \alpha \cos \beta \\
0 & \sin \alpha & \cos \alpha \cos \beta
\end{array}\right]\left[\begin{array}{c}
\dot{\alpha} \\
\dot{\beta} \\
\dot{\gamma}
\end{array}\right]
$$

The human body system consisting of $\mathrm{N}$ rigid bodies was solved by the second type of Lagrangian equation, as shown in formula 6 and 7, where $T$ is the kinetic energy of the $\mathrm{N}$ rigid bodies in the inertial system, expressed by the force $F_{i}$ and moments $M_{i}$ acting on the rigid body, $\mathrm{G}$ is the generalized vector, $m_{i}$ is the mass of the rigid body, and $I_{i}$ is the moment of inertia of the rigid body.

$$
\begin{gathered}
\frac{d}{d t}\left(\frac{\partial T}{\partial \dot{P}_{i}}\right)^{T}-\left(\frac{\partial T}{\partial P_{i}}\right)^{T}=G \\
T=\frac{1}{2} \sum_{i=1}^{N}\left(v_{i}^{T} m_{i} v_{i}+\theta_{i}^{T} I_{i} \theta_{i}\right)
\end{gathered}
$$

\subsection{D human body model}

The 3D human skeleton model, i.e., the virtual human model, is constructed by DIGuy, and imported into the virtual scene in FLT file format, which can be used for character shape selection, motion scheduling, motion path planning and real-time display, as shown in Fig.3 [23]. The constructed 3D virtual human body model realizes the modification of the configuration file and the calling of the model file through codes, and combines with $\mathrm{VC}++$ to develop the virtual athlete model interface of virtual reality sports and then realize the real-time display of the virtual human sports movement in the virtual reality system.

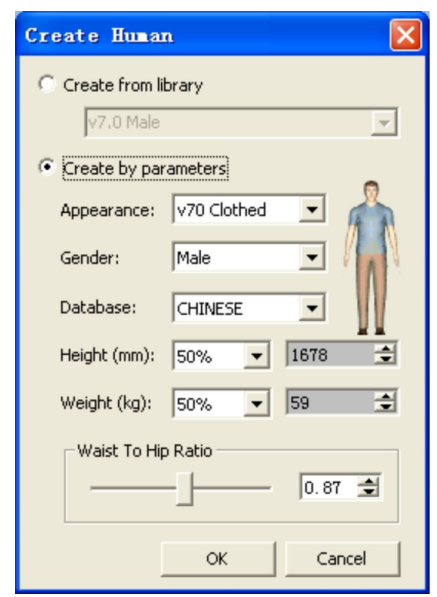

Fig. 3. Create human model 
The virtual human model is decomposed into various parts. The head and the body [24-26], the left and right arms and the body, the left/right legs and the body, the left/ right soles and the left/right calves [27-29], and the palm and the arm are all socketjointed; the first finger knuckle connected to the palm is a ball joint, and the connection between other finger knuckles is the cylindrical hinge joint [30]. Fig.4 shows its topology diagram, where the virtual human model is simplified to a multi-rigid geometry model, and constrained according to multi-rigid kinematics and dynamics theory.

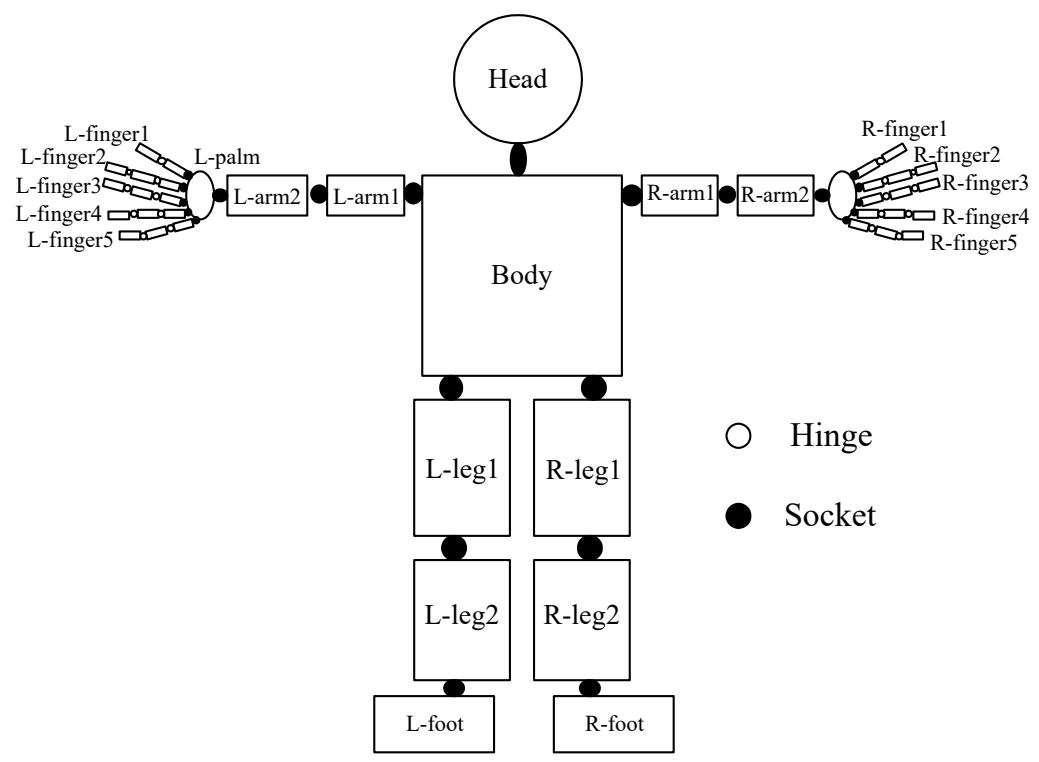

Fig. 4. Topological diagram of the connection between various parts of the human body

The black ball shown in Fig.4 indicates the socket-joint connection, while the white ball means the cylindrical hinge connection (mainly the connection between the finger joints), Combined with the ODE physics engine, the moving parts of the motion model are connected by the hinged constraint pair, so as to achieve the freedom of motion for each joint part, and constrain the range. The constraint equations of all hinge types are represented by formula 8 , where, $J$ and $\Omega$ are the Jacobian matrix $m \times 3, v_{1}, \theta_{1}$, and $v_{2}, \theta_{2}$ are the line velocity and angular velocity of the two connected objects respectively, $d$ is the right vector of the $m \times 1$-order, $\tau$ is the constraining force vector of $m \times 1$-order attached to the object for the establishment of the constraint equation and setting of the upper and lower limits, and $A$ is the $\mathrm{r}$ diagonal matrix of the $m \times m$-order, i.e., the mediation matrix of constraining force [31].

$$
\mathrm{J}_{1} v_{1}+\Omega_{1} \theta_{1}+J_{2} v_{2}+\Omega_{2} \theta_{2}=d+A \tau(l \leq \tau \leq h)
$$




\section{The Realization of Physical Education Motion Correction System}

\subsection{Capture device}

For the Kinect real-time motion capture device, its core technology is skeleton tracking. The infrared emitter, depth camera and infrared camera are hidden inside the casing, and the time-of-flight distance measurement technology is used to project the infrared light into the space, thereby calculating the phase difference of the infrared light and also the distance from each point in the space to the depth camera [32-37]. In order to ensure the accuracy of physical education motion correction, it is necessary to establish an all-dimensional stereoscopic display system. Therefore, multiple Kinect devices can be used to capture the sports movements at 360-degree through coordination in multi- angles, and the captured data can be synchronized to the computer in real time. Through computer calculation, it can be projected to the projection screen and displayed with 3D glasses in real-time. The design and implementation of the physical education teaching motion correction system based on computer virtual reality technology requires comparing and evaluating the sports movements, making the sports action correction more intuitive and targeted. In addition, the current realtime movement of the athlete should be compared with the standard movement, to display the gap between them in a visual form, and give the quantitative scores of the athlete's sports movement standard degree [38-45].

\subsection{Implementation and evaluation of virtual systems}

The technology device was used to measure, track and record the movement position of the athlete's various parts of the body during the movement, transforms the position into computer-recognizable data coordinates. Then, data analysis through the ordered logic program and algorithm was conducted, to generate the motion trajectory by the collected motion sequence. Finally, the real-time animation was generated by computer virtual reality technology, and can be played in a loop for physical education motion correction. Therefore, virtual motion can be described as a form of realtime spatial calculation based on computer technology.

The accuracy of the motion capture information depends on the setting of the motion capture point, that is, the number and position of the capture points, which is decisive for the accuracy of the 3D data recognition and the data setting of the postgenerated skeletal animation. For this, the motion relationship between the various parts of the human body, that is, the kinematics and dynamics data of the joint points was algorithmically debugged. Besides, combining the data model with the threedimensional skeleton model, the kinetic behavioural model of the human body was created, which was integrated into the PT virtual reality system through $\mathrm{VC}++$, ODE and API of Vega Prime. 
Table 1. Test score of related parameters between group A and B

\begin{tabular}{|l|c|c|c|c|c|c|c|}
\hline \multirow{2}{*}{ Category } & \multicolumn{2}{|c|}{ Group A } & \multicolumn{2}{c|}{ Group B } & Deviation of & t & 位 \\
\cline { 2 - 8 } & Mean value & $\begin{array}{c}\text { Standard } \\
\text { deviation }\end{array}$ & Mean value & $\begin{array}{c}\text { Standard } \\
\text { deviation }\end{array}$ & Mean value & value & p value \\
\hline Theory learning & 16.47 & 1.89 & 10.82 & 2.27 & 1.46 & 2.89 & $<0.05$ \\
\hline Sports action & 32.36 & 3.56 & 28.4 & 4.43 & 2.16 & 2.21 & $<0.05$ \\
\hline Accuracy & 33.27 & 3.02 & 30.18 & 4.12 & 2.26 & 2.44 & $<0.05$ \\
\hline
\end{tabular}

In this study, the experiment group A and control group B were set up. The targeted correction teaching based on virtual reality physical education was carried out for experimental group A, under the premise that the indicators of both groups had no significant difference. After the comparison experiment for a period of time, the related data were measured as shown in Table 1. Then, the mathematical statistics were made for the acquired data. It's concluded finally that the data of the experimental group A was significantly better than that of the control group B, and the difference was significant $(p<0.05)$, as shown in Fig.5. Meanwhile, the questionnaire was also conducted to evaluate and analyse the information obtained, and achieve the significant teaching feedback: $90 \%$ of students are interested in this kind of PE teaching mode based on computer virtual reality technology, thereby enhancing learning interest and motivation

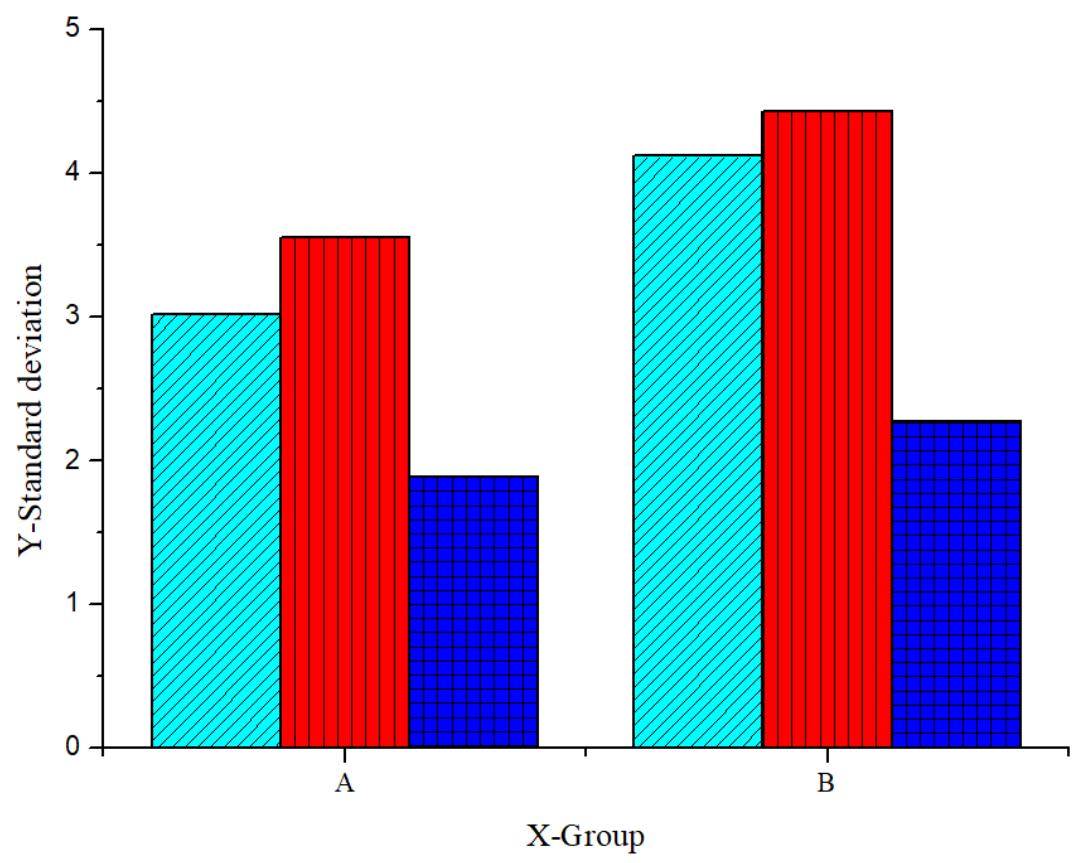

Fig. 5. Standard deviation comparison between group A and B 


\section{Conclusion}

The computer virtual reality technology is applied to the PE motion correction training, which can effectively solve the shortcomings of the traditional teaching mode, and correct the PE teaching actions in real time. Thus, the athletes can grasp the movement technical essentials as soon as possible and improve their training effect. The main research contents and conclusions are as follows:

- The virtual reality software Vega Prime was applied to create the hardware architecture of virtual reality system, analyse the virtual human skeleton structure by motion hinge topology, and simplify the human body into multi-rigid body system. For this, multi-rigid kinematics and dynamic equations are used to solve.

- The 3D human skeleton model, i.e., the virtual human model, was constructed by DI-Guy, and imported into the virtual scene in FLT file format. Then, combining with the skeleton structure and the joint motion law, the position data of the virtual human sports action could be obtained, and transmitted to the 3D human model in the virtual reality system through codes, so as to drive the virtual human model motion in real time.

- Using the computer virtual reality technology, the PE motion parameters and index parameters were obtained. Through analysis of real-time motion data and human sports movement posture, it can realize the real-time PE motion correction teaching and training in the $3 \mathrm{D}$ visualization manner.

- The PE teaching motion correction system based on computer virtual reality technology has obtained significant data advantages through comparative experiments, which provides a scientific basis for the evaluation of current PE teaching and training methods.

\section{$5 \quad$ References}

[1] Rothbaum, B.O., Hodges, L.F., Ready, D., Graap, K., Alarcon, R.D. (2001). Virtual reality exposure therapy for vietnam veterans with posttraumatic stress disorder. Journal of Clinical Psychiatry, 62(8): 617-622. http://doi.org/10.4088/JCP.v62n0808

[2] Franks, I.M., Nagelkerke, P. (1988). The use of computer interactive video in sport analysis. Ergonomics, 31(11): 1593-1603. http://doi.org/10.1080/00140138808966809

[3] Exadaktylos, K.A. (2004). Sports related maxillofacial injuries: the first maxillofacial trauma database in switzerland. British Journal of Sports Medicine, 38(6): 750-753. http://doi.org/10.1136/bjsm.2003.008581

[4] Aggarwal, R., Ward, J., Balasundaram, I., Sains, P., Athanasiou, T., Darzi, A. (2007). Proving the effectiveness of virtual reality simulation for training in laparoscopic surgery. Annals of Surgery, 246(5): 771-779. http://doi.org/10.1097/SLA.0b013e3180f61b09

[5] Angulo, R.M. (1992). Animation and analysis of sport events through 3d computer modelling. Journal of Biomechanics, 25(7): 761. http://doi.org/10.1016/00219290(92)90463-B

[6] Kitani, K. (2012). Ego-action analysis for first-person sports videos. IEEE Pervasive Computing, 92-95. http://doi.org/10.1109/MPRV.2012.28 
[7] Mcnamara, B.M. (1989). Relationship between computer resistance and worker characteristics in the sport industry: an exploratory analysis. Geophysics, 76(6): I59-I72. http://doi.org/10.1123/jsm.3.1.33

[8] Barris, S., Button, C. (2008). A review of vision-based motion analysis in sport. Sports Medicine, 38(12): 1025-1043. http://doi.org/10.2165/00007256-200838120-00006

[9] Wierzbicki, M., Drangova, M. (2004). Validation of dynamic heart models obtained using non-linear registration for virtual reality training, planning, and guidance of minimally invasive cardiac surgeries. Medical Image Analysis, 8(3): 387-401. http://doi.org/10.101 6/j.media.2004.06.014

[10] Cheung, K., Song, J., Yan, Q.S. (2007). Role of $h \rightarrow \eta \eta$ in Intermediate-Mass Higgs Boson Searches at the Large Hadron Collider. Physical Review Letters, 99(3): 031801. http://doi.org/10.1103/PhysRevLett.99.031801

[11] Hu, T., Lv, J., Xie, Q.S., Sun, H., Yuan, Q.N. (2017). A novel human behaviour information coding method based on eye-tracking technology. Traitement du Signal, 34(34), 153-173. https://doi.org/10.3166/TS.35.153-173

[12] Kelly, D. (2007). Development of a wearable motion capture suit and virtual reality biofeedback system for the instruction and analysis of sports rehabilitation exercises. 2007 29th Annual International Conference of the IEEE Engineering in Medicine and Biology Society, 2007(2): 4870-4874. http://doi.org/10.1109/IEMBS.2007.4353431

[13] Zhang, L. (2018). Research on human body movement posture based on inertial sensor, International Journal Bioautomation, 22(2), 179-186.

[14] Kirilova, K., Gatev, P. (2018). A complex semi-automatic method for kinetic and twodimensional kinematic motion analysis for posture and movement investigation, International Journal Bioautomation, 22(1), 57-64.

[15] Mentzelopoulos, M., Psarrou, A., Angelopoulou, A., José, G. (2013). Active foreground region extraction and tracking for sports video annotation. Neural Processing Letters, 37(1): 33-46. http://doi.org/10.1007/s11063-012-9267-4

[16] Jensen, J.E., Slocum, D.B., Larson, R. L., James, S.L., Singer, K.M. (1983). Reconstruction procedures for anterior cruciate ligament insufficiency: a computer analysis of clinical results. American Journal of Sports Medicine, 11(4): 240-248. http://doi.org/10.1177/ 036354658301100411

[17] Weber, P., Crispin, A., Schmidutz, F., Utzschneider, S., Pietschmann, M.F., Jansson, V. (2013). Improved accuracy in computer-assisted unicondylar knee arthroplasty: a metaanalysis. Knee Surgery Sports Traumatology Arthroscopy, 21(11): 2453-2461. http://doi.org/10.1007/s00167-013-2370-x

[18] Lassandro, P., Zonno, M. (2018). A work-related learning project for energy efficiency evaluation and indoor comfort of school buildings. Ingénierie des Systèmes d'Information, 23(5), 7-27. https://doi.org/10.3166/ISI.23.5.7-27

[19] Bai, Y. (2018). Design of human motion signal gathering system based on USB 2.0, International Journal Bioautomation, 22(S4), 325-336.

[20] Mirabella, O., Raucea, A., Fisichella, F., Gentile, L. (2011). A motion capture system for sport training and rehabilitation. International Conference on Human System Interactions, 52-59. http://doi.org/10.1109/HSI.2011.5937342

[21] Lim, C.H., Vats, E., Chan, C.S. (2015). Fuzzy human motion analysis: a review. Pattern Recognition, 48(5): 1773-1796. http://doi.org/10.1016/j.patcog.2014.11.016

[22] Bejek, Z., Paróczai, R., Szendri, M., Kiss, R.M. (2011). Gait analysis following TKA: comparison of conventional technique, computer-assisted navigation and minimally invasive technique combined with computer-assisted navigation. Knee Surgery Sports Traumatology Arthroscopy, 19(2): 285-291. http://doi.org/10.1007/s00167-010-1219-9 
[23] Zhou, M., Tan, G., Zhong, Z., Hu, F. (2009). Design and Implementation of a Virtual Indoor Roaming System Based on Web3D. First International Workshop on Education Technology \& Computer Science. IEEE Computer Society, 985-988. http://doi.org/10. $\underline{1109 / \text { ETCS.2009.503 }}$

[24] De Loor, P., Richard, R., Bevacqua, E. (2018). Evolutive body interaction between a human and a virtual character. Theoretical model proposition and evaluation within a fitness exergame, Revue d'Intelligence Artificielle, 31(5), 557-579. https://doi.org/10. 3166/RIA.31.557-579

[25] Bricken, M., Byrne, C.M. (1993). Summer students in virtual reality: a pilot study on educational applications of virtual reality technology. Virtual Reality, 199-217.

[26] Rose, F.D., Attree, E.A., Johnson, D.A. (1996). Virtual reality: an assistive technology in neurological rehabilitation. Current Opinion in Neurology, 9(6): 461. http://doi.org/10.1097/00019052-199612000-00012

[27] Vora, J., Nair, S., Gramopadhye, A.K., Duchowski, A.T., Melloy, B.J., Kanki, B. (2002). Using virtual reality technology for aircraft visual inspection training: presence and comparison studies. Applied Ergonomics, 33(6): 559-570. http://doi.org/10.1016/S0003$\underline{6870(02) 00039-X}$

[28] Eastgate, R.M., Griffiths, G.D., Waddingham, P.E., Moody, A.D., Butler, T.K.H., Cobb, S. V. (2005). Modified virtual reality technology for treatment of amblyopia. Eye (Basingstoke): 20(3): 370-374. http://doi.org/10.1038/sj.eye.6701882

[29] Judith, A.B.P.D. (2004). Experience with virtual reality-based technology in teaching restorative dental procedures. Journal of Dental Education, 68(12): 1258-1265.

[30] Yang, J.C., Chen, C.H., Jeng, M.C. (2010). Integrating video-capture virtual reality technology into a physically interactive learning environment for English learning. Computers and Education, 55(3): 1346-1356. http://doi.org/10.10 16/j.compedu.2010.06.005

[31] Zhang, L., Liu, Q. (2012). Application of Simulation and Virtual Reality to Physical Education and Athletic Training. Transactions on Edutainment VII, 24-33. http://doi.org/10.1007/978-3-642-29050-3 3

[32] Watanuki, K. (2010). Development of virtual reality-based universal design review system. Journal of Mechanical Science \& Technology, 24(1): 257-262.

[33] Durand-Texte, T., Simonetto, E., Durand, S., Melon, M., Moulet, M.H. (2017). Estimation of the uncertainties of a method of measuring vibration deformations by $3 \mathrm{D}$ vision, Instrumentation Mesure Métrologie, 16(1-4), 71-95. https://doi.org/10.3166/i2m.16.1-4.7195

[34] Stark, R., Israel, J.H., Whler, T. (2010). Towards hybrid modelling environments-merging desktop-cad and virtual reality-technologies. CIRP Annals - Manufacturing Technology, 59(1): 179-182. http://doi.org/10.1016/j.cirp.2010.03.102

[35] Sun, F., Zhang, Z.C., Liao, D.M., Chen, T., Zhou, J.X. (2015). A lightweight and crossplatform web3d system for casting process based on virtual reality technology using webgl. The International Journal of Advanced Manufacturing Technology, 80(5-8): 801816. http://doi.org/10.1007/s00170-015-7050-1

[36] Saposnik, G., Bayley, M., Cheung, D., Willems, J., Mamdani, M., Cohen, L. (2009). Fp12mo-04 virtual reality technology in stroke rehabilitation: a pilot randomized trial using WII gaming system. Journal of the Neurological Sciences, 285(09): S76-S76. http://doi.org/10.1016/S0022-510X(09)70326-0

[37] Curley, K.F. (1989). Computer technology and knowledge workers: a pilot study of job impact. Interacting with Computers, 1(2): 171-182. http://doi.org/10.1016/0953$\underline{5438(89) 90024-6}$ 
[38] Soc, I.C. (1981). Computer technology moves fast. Computer, 14(5): 107-110. http://doi.org/10.1109/C-M.1981.220392

[39] Greenstein, S. (1995). The market and computer technology. IEEE Micro, 15(3): 8-9, 78. http://doi.org/10.1109/40.387675

[40] Myers, W. (1976). Key developments in computer technology: a survey. Computer, 9(11): 48-77. http://doi.org/10.1109/C-M.1976.218441

[41] Burger, R.M., Cavin, R.K.I., Holton, W.C., Sumney, L.W. (1984). The impact of ICS on computer technology. Computer, 17(10): 88-95. http://doi.org/10.1109/MC.1984.1658961

[42] Steier, L.P. (1986). Computer technology and education: a policy delphi. Computer Software, 1-29.

[43] Hardy, J.V. (1998). Teacher attitudes toward and knowledge of computer technology. Computers in the Schools, 14(3-4): 119-136. http://doi.org/10.1300/J025v14n03 11

[44] Weber, R. (1988). Computer technology and jobs: an impact assessment model. Communications of the ACM, 31(1): 68-77. http://doi.org/10.1145/35043.35049

[45] Blachman, N.M. (1961). The state of digital computer technology in Europe. Communications of the Acm, 4(6): 256-267. http://doi.org/10.1145/366573.366596

\section{Authors}

Yongzhong Yang is a physical education teacher of Yangtze Normal University, was born in June 1981. He works in Fuling, Chongqing. He graduated from Beijing Sports University and was a postgraduate from Chongqing University. He is mainly engaged in traditional ethnic sports and commits himself to sports medicine research. He has published a paper in Beijing Spots University and two papers in Chinese core journals.

Lingfeng Meng graduated from Hebei Normal University with master degree, has undertaken and participated in 3 province departmental level topics and published 3 core journal papers. The research field is physical education with the title of lecturer.

Article submitted 2019-04-24. Resubmitted 2019-05-29. Final acceptance 2019-06-03. Final version published as submitted by the authors. 